\title{
Operación del sistema de autocontrol y gestión del riesgo de lavado de activos y financiación del terrorismo en empresas del sector comercial
}

\section{Operation of the system for self-control and management of the risk of money laundering and financing of terrorism in companies of the commercial sector}

\author{
Eduardo Alexander Duque Grisales ${ }^{a}$, Jeniffer Molina Flórez ${ }^{b}$, \& Nataly Ossa Núñez c \\ a Institución Universitaria ESUMER, Medellín, Colombia. eduardo.duque@esumer.edu.co \\ b Institución Universitaria ESUMER, Medellín, Colombia. jeniffer.molina@esumer.edu.co \\ a Institución Universitaria ESUMER, Medellín, Colombia.nataly.ossa@esumer.edu.co
}

Recibido: mayo 29, 2018. Aceptado: junio 20, 2018.

\begin{abstract}
Resumen
El lavado de activos y la financiación del terrorismo (LA/FT) se consideran como una gran amenaza para la economía mundial ya que afectan los indicadores internos, la integridad de los mercados y definitivamente la globalización e intercambio comercial en condiciones transparentes y confiables. Tales afectaciones han sido consideradas por entes gubernamentales y entidades de carácter global que buscan a través de diversas normas combatir la inclusión de los recursos económicos de dichos fenómenos delictivos en la economía. Las empresas del sector comercial en Colombia no son ajenas a esta realidad y han venido experimentando un constante riesgo de inclusión de recursos procedentes de actividades ilícitas. Por lo anterior, resulta necesario y conveniente que estas empresas se protejan contra estos riesgos por medio de la implementación del Sistema de Autocontrol y Gestión del Riesgo de LA/FT - SAGRLAFT, definido por la Superintendencia de Sociedades, a través de los lineamientos impartidos en la Circular externa 100-000006 del 19 de agosto del 2016, Capítulo X. En este artículo se presenta un procedimiento resumido para que las empresas del sector comercial implementen medidas preventivas para dar frente al LA/FT. Con la implementación del SGRLAFT se reduce el riesgo de incurrir en sanciones legales, se evita la complicidad y corrupción con organizaciones criminales y/o ilegales y se mejora la reputación empresarial.
\end{abstract}

Palabras Clave: lavado de activos, financiación del terrorismo, Sistema de Autocontrol y Gestión del Riesgo de LA/FT, Administración del riesgo.

\begin{abstract}
Money laundering and the financing of terrorism (ML/FT) are seen as significant threats to the global economy as they affect internal indicators, the integrity of markets, and ultimately the worldwide trade in clear and reliable conditions. Governmental and global business entities have faced such affectations through different norms to fight the inclusion of the economic resources of these criminal phenomena in the world economy. Companies in the commercial sector in Colombia are not exempt from this reality, as they have been experiencing a constant risk of including resources from illicit activities. Therefore, it is necessary and convenient that these companies protect themselves against these risks through the implementation of the code for Self-Control and Risk Management System of Money Laundering and financing of terrorism, also known as SAGRLAFT. This law is defined by the Superintendence of Companies, through the guidelines provided in the External Circular 100-000006 of August 19, 2016, Chapter X. This article presents a summary procedure for companies in the commercial sector to implement preventive measures from SAGRLAFT to deal with money laundering and financing of terrorism. The implementation of these preventive policies reduces the risk of incurring in legal sanctions. Also, companies avoid suspicions of corruption with criminal or illegal organizations, and business reputation is improved.
\end{abstract}

Keywords: Money laundering, financing of terrorism, Self-Control and Risk Management System of ML/FT, Risk management.

\section{Citar como:}

E. Duque-Grisales, J. Molina, N. Ossa “Operación del sistema de autocontrol y gestión del riesgo de lavado de activos y financiación del terrorismo en empresas del sector comercial” Revista CINTEX, Vol. 23(1), pp. 32-42. 2018. 


\section{INTRODUCCIÓN}

La prevención y el control del lavado de activos y de la financiación del terrorismo - LA/FT se han asumido, en las últimas décadas, con mayor importancia en el contexto nacional e internacional debido a que se ha evidenciado que estos fenómenos sociales afectan negativamente la economía de un país [1], [2]; debido a ello, las empresas optan por implementar sistemas con los cuales logren cumplir dicho objetivo y de esta forma evitar ser utilizados con el fin de ofrecer apariencia de legalidad a los recursos obtenidos de manera ilícita.

Debido a que el lavado de dinero y el financiamiento del terrorismo involucran el desarrollo de actividades criminales y procedencia indebida de los recursos, es necesario establecer normativas ajustadas a cada sector económico para evitar esta realidad [3]. Algunas instituciones internacionales, como el Grupo de Acción Financiera -GAFI- proporcionan un conjunto completo de contramedidas contra el LA/FT que cubren el sistema de justicia penal y la aplicación de la ley, el sistema financiero y su regulación y la cooperación internacional. Las Recomendaciones del GAFI, aunque no representan una convención internacional vinculante, han sido reconocidas, respaldadas o adoptadas por más de 182 países y jurisdicciones que han asumido un compromiso político para combatir el lavado de dinero y el financiamiento del terrorismo al implementar los estándares del GAFI [4] - [7]. Las normas establecen los principios de acción de los gobiernos y las autoridades competentes y permiten a los países una cierta flexibilidad en la implementación de estos principios de acuerdo con sus circunstancias únicas y marcos constitucionales [8], [9].

En el caso de Colombia, el sector financiero fue el primero en implementar un sistema de administración del riesgo del LA/FT; a partir del 2014, la Superintendencia de Sociedades a través de la Circular Externa 304-000001 impartió la instrucción de implementar el SAGRLAFT en algunas empresas del sector real para seguir las recomendaciones del GAFI y ampliar los sectores del país en los que se adoptan sistemas para prevenir y controlar este tipo de actividades delictivas. A dicha Circular se le han realizado algunos ajustes, los más recientes se mencionan en la Circular Externa 100-000006 del 19 de agosto de 2016, Capítulo X [23]. De acuerdo con la Superintendencia de Sociedades [10], cada empresa debe analizar su exposición al riesgo teniendo en cuenta las normas, lineamientos y estándares internacionales presentados en dicha circular y debe diseñar e implementar el SAGRLAFT según sus propias necesidades (clientes, proveedores, empleados, jurisdicciones, canales de venta, políticas de dinero en efectivo, entre otros factores).

Las empresas del sector comercial en Colombia han corrido el riesgo de vincular dineros de procedencia ilícita en los últimos años, dado sus bajos niveles de innovación tecnológica [11], capacidad productiva [12] y, especialmente, necesidades de capital para financiar su operación, razón por la cual se espera generar un mayor control de sus procesos de financiación y la implementación de sistemas de control efectivo para prevenir este fenómeno y reducir el impacto de cualquier actividad criminal. El objetivo de este trabajo es brindar un procedimiento claro para la implementación del SAGRLAFT en las empresas del sector comercial que se encuentran obligadas a reportar ante la Unidad de Investigación y Análisis Financiero (UIAF) los mecanismos adoptados para la prevención y el control del LA/FT, para tal fin se realiza una revisión bibliográfica extensa a partir del análisis de las recomendaciones del GAFI y la normativa exigida por la Superintendencia Financiera de Colombia para la implementación del SARLAFT. La aplicación de este tipo de sistemas de control del LA/FT mejoran la gestión del riesgo de las empresas del sector comercial, garantizan seguridad y confianza para los inversionistas, clientes y demás partes interesadas de la empresa.

El resto del artículo está organizado de la siguiente manera. En el capítulo 2 se presenta una revisión de literatura sobre el LA/FT. En el capítulo 3 se presenta la metodología utilizada en este artículo para definir el procedimiento adecuado de implementación del SARLAFT en las empresas del sector comercial. Posteriormente, se presentan el análisis de resultados y las conclusiones.

\section{REVISIÓN DE LITERATURA}

Varios autores han discutido el uso de sistemas de gestión de riesgos de recursos para financiación del terrorismo y el lavado o blanqueamiento de activos en entidades comerciales de diferentes sectores financieros [13]- [15], dado que este tipo de actividades ilegales constituyen un peligro latente en la operación de empresas en todos los rincones de la economía internacional, y, particularmente, para las actividades financieras en Colombia. En este último país, el organismo encargado de la vigilancia de este tipo de situaciones es la Superintendencia Financiera de Colombia, la cual define los riesgos de Lavado de Activos y Financiación del Terrorismo (LA/FT) en la Circular Externa Nro. 007 [16], según la cual se pueden entender como: "La posibilidad de pérdida o daño, que puede sufrir una entidad vigilada por su propensión a ser utilizada directamente o a través de sus operaciones, como instrumento para el lavado de activos y/o canalización de recursos hacia la realización de actividades terroristas, o cuando se pretenda el ocultamiento de activos provenientes de dichas actividades". 
Operación del sistema de autocontrol y gestión del riesgo de lavado de activos y financiación del terrorismo en empresas del sector comercial

Operation of the system for self-control and management of the risk of money laundering and financing of terrorism in companies of the commercial sector

Prácticamente todos aspectos de la economía en Colombia se ven afectados por los problemas de LA/FT, pues dado que las operaciones financieras están siendo vigiladas con especial detalle para evitar este tipo de actividades, la delincuencia se ha enfocado en la creación de entes empresariales "reales" para ejecutar las maniobras de LA/FT. Con respecto a esta situación, el experto Mauricio Montoya opinaba que "las empresas criminales y las operaciones de financiamiento del terrorismo tienen éxito en buena medida, si es que consiguen blanquear y encubrir sus ganancias transfiriéndolas a través de sistemas financieros nacionales e internacionales" [17]. Los blanqueadores de dinero y los financiadores del terrorismo utilizan diferentes técnicas de colocación, estratificación e integración. Las sumas de dinero involucradas en el LA/FT varían significativamente [18] - [21]. De acuerdo con un estudio realizado por el Fondo Monetario Internacional, se estima que las organizaciones dedicadas al blanqueo de capitales logran una utilidad neta equivalente al $3 \%$ del producto interno bruto mundial y aproximadamente el $25 \%$ de las actividades ilícitas que se presentan alrededor del mundo corresponden al blanqueo de capitales [22].

La revista Infolaft, Anticorrupción, Fraude y LA/FT tiene como uno de sus objetivos informar al público en general de todos los acontecimientos, cambios, novedades y demás datos relacionados con el LA/FT en Colombia y el mundo. En su edición 82 de enero de 2016 "Las sanciones por incumplimientos en norma LA/FT llegarían este año" plantea que la Superintendencia de Sociedades envió un oficio el 09 de abril de 2015 a todas las empresas del sector real comunicando la obligación de implementación del "Sistema de Autocontrol y Gestión del Riesgo de Lavado de Activos y Financiación del Terrorismo" (SAGRLAFT), con el cual pretendía obtener información de los avances que tenía cada una de ellas en lo referente a los planes de prevención de LA/FT. Las empresas que no lo respondieron oportunamente deberán explicar las razones de su incumplimiento, las cuales serán analizadas por Supersociedades y, de acuerdo con la justificación, se iniciaría con el pliego de cargos correspondiente; por otra parte, en el artículo publicado el 25 de julio de 2016 "Supersociedades oficializa propuesta para ampliar sectores obligados a prevenir el lavado" informa que la Superintendencia de Sociedades presentó una propuesta para modificar el capítulo 10 de la circular básica jurídica con el fin de obligar a más empresas del sector real a implementar el SAGRLAFT, las cuales tienen montos determinados de acuerdo con el sector, entre ellas se encuentran: empresas del sector inmobiliario, dedicadas a la explotación de minas, de servicios jurídicos, de servicios contables, de comercio de vehículos y de autopartes, y de construcción de edificios; mientras que en el artículo publicado el 08 de agosto de 2016 "La realidad penal del lavado de activos en Colombia" revela que en el 2017 el Fondo Monetario Internacional evaluará los resultados y la efectividad del sistema adoptado en Colombia contra el lavado de activos y la financiación del terrorismo, así como la cantidad y calidad de las condenas que se han emitido a quienes han cometido dichos delitos. En la publicación se enuncia que el narcotráfico y el enriquecimiento ilícito son las actividades que mayores ingresos les generan a los lavadores, quienes son condenados en promedio a 100 meses de cárcel (8,3 años), el proceso de condena tiene una duración aproximada de 59 meses (casi 5 años) y resalta que entre el 2005 y el 2015 se emitieron 1,157 condenas.

EI SAGRLAFT se convierte en parte integral de la gestión de riesgos que implementan las sociedades para prevenir, mitigar y evitar que se materialicen eventos de riesgo a los cuales se ven expuestas cotidianamente, la falta de conocimiento de la norma y su interpretación inadecuada se convierten en factores que impiden identificar la metodología apropiada para prevenir y controlar la materialización de eventos de riesgo relacionados con el LA/FT. Teniendo en cuenta las características del SAGRLAFT, también se debe tener presente que su adopción debe cumplir con los parámetros descritos en la figura 1, los cuales se deberán ajustar de acuerdo con las características propias de cada empresa (tamaño, productos, canales, jurisadisaipenérstętdelncia de Sociedades, a través de su última actualización promovida en la Circular Externa 100-000006 de 2016 [23], obliga a poner en práctica el Sistema de Autocontrol y gestión del riesgo de LA/FT

a: Empresas que están bajo su inspección y vigilancia.

- Empresas del "sector inmobiliario, sector de explotación de minas y canteras, sector de servicios jurídicos, sector de servicios contables, de cobranza y/o calificación crediticia, sector de comercio de vehículos; sus partes, piezas y accesorios, sector de construcción de edificios" [23].

- Empresas que no hacen parte de dichos sectores pero que están bajo su inspección y vigilancia y que "al 31 de diciembre del año inmediatamente anterior hayan obtenido ingresos totales iguales o superiores a 160,000 SMMLV (Salario Mínimo Legal Vigente)" [23].

- El plazo para implementar, diseñar o ajustar el SAGRLAFT es de doce meses.

Al adoptar políticas del SAGRLAFT es importante implementar la debida diligencia ya que esta metodología permite la reducción de las probabilidades de consideración de culpabilidad por omisión, con las correspondientes 
consecuencias penales, administrativas y civiles del caso. Por lo tanto, es necesario tener en cuenta que se debe elaborar el reporte de operación sospechosa (ROS) ante la UIAF, incluyendo la verificación de identidad tanto del beneficiario final como del cliente, para cada operación o transacción ocasional o durante cualquier momento de una relación comercial, siempre que una entidad empresarial tenga sospechas de posibles actividades de LA/FT.

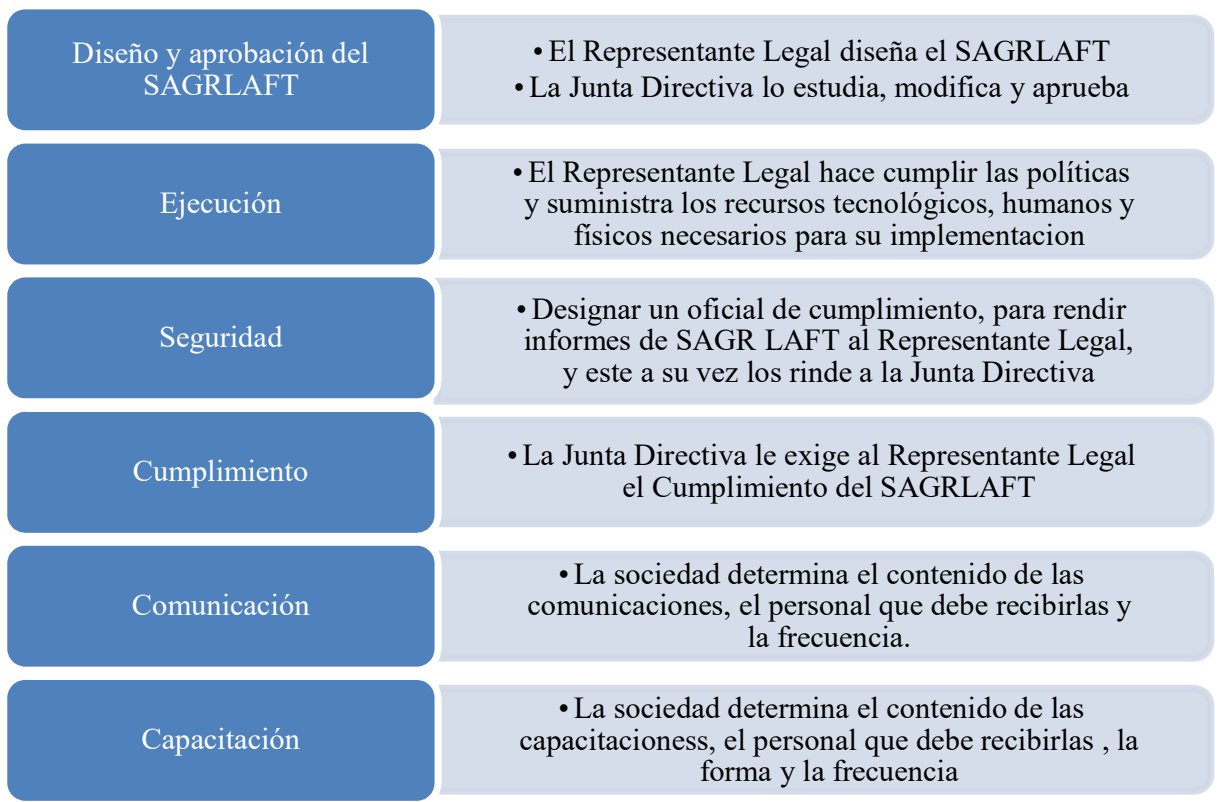

Figura 1. Parámetros del SAGRLAFT [23]

\section{METODOLOGÍA}

Para el desarrollo de esta investigación se empleará una metodología documental y comparativa que facilite entender los requerimientos exigidos en el SAGRLAFT bajo los cuales operan las empresas del sector comercial. Teniendo en cuenta que el SAGRLAFT es un nuevo sistema de implementación en el sector real, la investigación se basa inicialmente en el estudio e interpretación de la Circular externa 100-000006 del 19 de agosto del 2016, Capítulo X; posteriormente, se toman como precedentes las investigaciones elaboradas en diferentes artículos y noticias publicadas sobre el tema como referentes de la actualidad nacional e internacional frente al control y prevención de LA/FT.

De acuerdo con la Circular Externa 100-00006 del 2016, Capítulo X, las entidades bajo la vigilancia de la Superintendencia de Sociedades que a 31 de diciembre del año inmediatamente anterior hayan alcanzado ingresos totales iguales o superiores a 160,000 SMMLV tienen la obligación de adoptar el SAGRLAFT y enviar los reportes correspondientes durante el año siguiente a la UIAF (cada año se realiza el mismo cálculo para todas las empresas del sector y se deben cumplir las mismas condiciones) y su incumplimiento incurre en sanciones de tipo administrativo y legal.

EI SAGRLAFT se convierte en parte integral de la gestión de riesgos que implementan las sociedades para prevenir, mitigar y evitar que se materialicen eventos de riesgo a los cuales se ven expuestas cotidianamente, la falta de conocimiento de la norma y su interpretación inadecuada se convierten en factores que impiden identificar la metodología apropiada para prevenir y controlar la materialización de eventos de riesgo relacionados con el LA/FT. Por consiguiente, atendiendo a intereses de innovación en los procesos de gestión empresarial [24], y con el fin de acoger las normas internacionales, cumplir sus objetivos, mantener la economía sana y teniendo en cuenta el desconocimiento que existe en el sector comercial sobre el SAGRLAFT, se pretende ofrecer una guía que les permita a las sociedades adoptar dicho sistema de forma clara y efectiva siguiendo los lineamientos mencionados en la Circular Externa 100-000006 del 19 de agosto de 2016, Capítulo X [23].

El Grupo de Acción Financiera Internacional - GAFI, agrupación intergubernamental que busca el desarrollo y la promoción de políticas para la protección del sistema financiero global de operaciones de lavado de 
Operación del sistema de autocontrol y gestión del riesgo de lavado de activos y financiación del terrorismo en empresas del sector comercial

Operation of the system for self-control and management of the risk of money laundering and financing of terrorism in companies of the commercial sector

capitales, el apoyo económico para la proliferación de armas de destrucción masiva y las operaciones de financiamiento del terrorismo, sugirió que se adoptaran las medidas correspondientes con las características inherentes a los riesgos señalados y, en febrero de 2012, diseñó 40 estatutos de validez internacional acerca de los problemas que busca combatir, entre las cuales se destacan:

- Recomendación 1: evaluación de riesgos y aplicación de un enfoque basado en riesgo.

- $\quad$ Recomendación 28: regulación y supervisión de las APNFD (actividades y profesiones no

financieras designadas) operativo y orden público.

- Recomendación 34: guía y retroalimentación.

- Recomendación 35: sanciones

De acuerdo con el GAFI, para obtener los resultados esperados del sistema de autocontrol y gestión del riesgo LA/FT se deben asegurar sanciones efectivas, ya sean administrativas, civiles o penales, las cuales deben ser aplicables para personas naturales (incluye a directivos y a la alta gerencia) o personas jurídicas que incumplan con las medidas para combatir el LA/FT.

\section{ANÁLISIS DE RESULTADOS: ETAPAS DE EJECUCIÓN DEL SAGRLAFT}

Para el diseño, elaboración y cumplimiento del SAGRLAFT se deben desarrollar las recomendaciones impartidas por la Superintendencia de Sociedades a través de la Circular Externa 100-000006 de 2016 [23]. A continuación, se recomiendan los pasos que deben seguir las empresas del sector comercial obligadas a implementar el SAGRLAFT, los cuales le permitirán cumplir con los lineamientos impartidos en la Circular y facilitarán su adopción al interior de la compañía.

\subsection{ETAPA 1: DIAGNÓSTICO}

Consiste en comprometer a los directores y a la alta gerencia de la empresa en la implementación del SAGRLAFT con la definición de metodologías, técnicas y herramientas de acuerdo con las características de la empresa, teniendo en cuenta las diferentes fuentes de información para su diseño y adopción.

- PASO 1: concientizar a directores y a la alta gerencia de la empresa en la importancia de la adopción y el fortalecimiento de medidas para la prevención del SAGRLAFT, lo cual requiere de la elaboración de códigos de ética, manuales y procedimientos.

- PASO 2: identificar y analizar las características internas y externas de la empresa. El análisis de las características internas comprende:

- El direccionamiento estratégico (visión, misión, debilidades, oportunidades,

fortalezas y amenazas).

$\circ \quad$ El entorno interno de los negocios (el desarrollo del objeto social).

Los interesados internos (quienes se deben involucrar en el SAGRLAFT).

Definir los objetivos de la administración de riesgos de LA/FT.

Definir la finalidad del SAGRLAFT en la empresa.

Definir las políticas de la administración de riesgos de LA/FT, las cuales deben

permitir el eficiente, efectivo y oportuno funcionamiento del LA/FT.

○ Definir la estructura organizacional: según la Circular Externa 100-000006 de 2016

[23], la estructura organizacional debe estar conformada mínimamente por la Junta Directiva, el representante legal y el oficial de cumplimiento.

- Definir el sistema de control interno: el oficial de cumplimiento debe designar los responsables del SAGRLAFT en la empresa.

- PASO 3: determinar los factores de riesgo del LA/FT. Los factores de riesgo del LA/FT deben ser objeto de administración y control, para una empresa comercial los factores de riesgo mínimo son:

- Las contrapartes: personas naturales o jurídicas con quienes la empresa tiene relación comercial.

- Los productos: son las operaciones realizadas por la empresa en desarrollo de su objeto social.

- Los canales de distribución: son los canales o medios que utiliza la empresa para la distribución de los productos. Las jurisdicciones: regiones donde la empresa desarrolla su actividad económica o donde le cliente se encuentra ubicado. 
- PASO 4: elaboración del diagnóstico del riesgo de LA/FT. El diagnóstico de LA/FT debe reflejar los factores de riesgo y debe estar basado en determinar una aproximación al nivel de riesgo inherente del LA/FT de la empresa, con base en este diagnóstico se desarrollan las etapas del SAGRLAFT (identificación, medición, control y monitoreo).

- PASO 5: definir las metodologías y herramientas para la gestión del riesgo LA/FT. En este paso es necesario definir las metodologías que permitan la identificación, segmentación de los factores de riesgo y su clasificación. Adicionalmente, se debe establecer la herramienta que se utilizará para parametrizar las señales de alerta, con las cuales se logra detectar oportunamente las operaciones inusuales para su respectivo análisis.

\subsection{ETAPA 2: IDENTIFICACIÓN DE LOS RIESGOS}

Consiste en identificar los eventos de riesgo de LA/FT inherentes al desarrollo del objeto social de la empresa en cada uno de los factores, para lo cual la empresa se debe basar en:

- Las matrices de riesgo existentes o proceder en su diseño.

- El nivel de riesgo de la empresa.

- Su tamaño y el desarrollo de los sistemas de gestión de riesgo.

Además, se debe elaborar una lista de los posibles eventos de riesgo (fuentes internas o externas generadoras de un riesgo asociado al LA/FT). La lista permite definir qué puede suceder si dichos eventos se presentan, y se debe basar en el análisis interno utilizando el análisis de tipologías, señales de alerta, documentos y recomendaciones internacionales sobre prevención del LA/FT. En la tabla 1 se muestra el formato a utilizar para la identificación de los riesgos asociados con el lavado de activos y la prevención del terrorismo LA/FT

Tabla 1. Formato para la identificación de riesgos

\begin{tabular}{|c|c|c|}
\hline TABLE I. CODDIGO & $\begin{array}{l}\text { TABLE II. EVENTO } \\
\text { DE RIESGO }\end{array}$ & TABLE III. CAUSA \\
\hline $\begin{array}{l}\text { TABLE IV. DETERMINAR UN } \text { CóDIGO } \\
\text { INTERNO QUE PERMITA LA IDENTIFICACIÓN DEL } \\
\text { EVENTO DE RIESGO }\end{array}$ & $\begin{array}{l}\text { TABLE V. ¿QUÉ PUEDE } \\
\text { SUCEDER? }\end{array}$ & $\begin{array}{cc}\text { TABLE VI. } & \text { ¿CÓMO } \\
\text { POR QUÉ PUEDE SUCEDER? }\end{array}$ \\
\hline
\end{tabular}

\subsection{ETAPA 3: MEDICIÓN O EVALUACIÓN DE RIESGOS}

Consiste en medir la probabilidad de ocurrencia de riesgos inherentes de cada uno de los eventos de riesgo, así como el impacto en caso de materializarse mediante los riesgos asociados. Para calcular el nivel de riesgos se deben combinar las posibilidades y las consecuencias.

- PASO 1: identificar los criterios para la medición de los riesgos.

- Probabilidad: es la posibilidad de que un riesgo se materialice, para lo cual la empresa establece los nombres y cantidad de rangos que utilizará, por ejemplo, se pueden utilizar los siguientes:

- Muy Probable: Alta probabilidad de ocurrencia.

- Posible: media probabilidad de ocurrencia.

- Raro: baja probabilidad de ocurrencia

- Impacto: son los daños o efectos que puede sufrir la empresa en caso de que se materialice un evento de riesgo. La empresa puede definir los rangos que utilizará de acuerdo con el nivel de riesgo que aceptará; generalmente se utilizan mínimo tres rangos, al igual que puede definir los nombres de cada rango, por ejemplo, bajo, medio y alto. En la tabla 2 se muestra el cálculo de la matriz de probabilidades e impacto de los riesgos asociados con el lavado de activos y la prevención del terrorismo LA/FT

- PASO 2: Procedimientos para la medición del riesgo de LA/FT. Posterior a la clasificación de los riesgos y la identificación de probabilidades e impactos se debe:

- Realizar un plan de tratamiento que permita calificar los eventos de riesgo, con el fin de que sean objeto de los procedimientos y controles para la administración o mitigación del riesgo.

- Generar la matriz de riesgo por cada factor de riesgo 
Operación del sistema de autocontrol y gestión del riesgo de lavado de activos y financiación del terrorismo en empresas del sector comercial Operation of the system for self-control and management of the risk of money laundering and financing of terrorism in companies of the commercial sector

Tabla 2. Matriz posibilidad vs impacto

\begin{tabular}{|l|l|l|l|}
\hline $\begin{array}{l}\text { PROBABILIDAD } \\
\text { IMPACTO }\end{array}$ & \multicolumn{2}{l|}{ 1-BAJO } & 2-ALTO \\
\hline A - MUY PROBABLE & $1-\mathrm{A}$ & $2-\mathrm{A}$ & $3-\mathrm{A}$ \\
\hline B - POSIBLE & $1-\mathrm{B}$ & $2-\mathrm{B}$ & $3-\mathrm{B}$ \\
\hline C - RARO & $1-\mathrm{C}$ & $2-\mathrm{C}$ & $3-\mathrm{C}$ \\
\hline
\end{tabular}

\subsection{ETAPA 4: CONTROL}

El control es una disminución de la posibilidad de ocurrencia o del impacto del riesgo de LA/FT en caso de materializarse. Para dar funcionalidad a esta etapa se deben definir las metodologías y procedimientos con el fin de adoptar los controles que permitan detectar operaciones inusuales o sospechosas. En esta etapa se deben realizar los siguientes pasos:

- PASO 1: definir los controles para mitigar los eventos de riesgo. En la tabla 3 se presenta el formato utilizado para la identificación de los riesgos asociados con el lavado de activos y la prevención del terrorismo LA/FT, y la descripción del control que se debe realizar.

Tabla 3. Formato para la identificación de eventos de riesgo

\begin{tabular}{|c|c|cc|}
\hline CÓDIGO & EVENTO DE RIESGO & \multicolumn{2}{c|}{ DESCRIPCION DE LOS } \\
CONTROLES REQUERIDOS
\end{tabular}

- PASO 2: definir los procedimientos para la aplicación de los controles. Es necesario definir para cada factor de riesgo LA/FT los procedimientos para la prevención del LA/FT. En cada proceso se deben describir los procedimientos y los controles efectivos que se implementarán para detectar las operaciones inusuales y sospechosas. También se requiere definir los controles para la segmentación de las contrapartes por niveles de riesgo (al momento de su vinculación), controlar y monitorear las operaciones de las contrapartes y detectar las operaciones inusuales.

- PASO 3: diseñar y aplicar un plan de tratamiento de los riesgos de LA/FT. La empresa debe diseñar y ejecutar un plan de tratamiento en la respectiva matriz de riesgo, el cual consiste en:

- Describir las acciones que se ejecutarán: definición y características del tratamiento.

Priorizar las medidas que se adoptarán: alta, media o baja.

Definir el(los) responsables de la implementación del plan.

Presupuestar los recursos económicos para la ejecución del plan.

Definir el responsable del monitoreo.

Elaborar el cronograma de la implementación

Monitorear el plan propuesto

- PASO 4: seguimiento y control de las operaciones de las contrapartes. Las empresas tienen la obligación de detectar operaciones inusuales y reportar operaciones sospechosas de las contrapartes, por lo cual es necesario adoptar un sistema de control de las operaciones de sus "contrapartes" teniendo en cuenta los principios básicos dentro del desarrollo de los procedimientos.

- Principio "conozca a su contraparte": este principio está basado en el conocimiento de la contraparte, origen y destino de sus recursos económicos, así como sus antecedentes, para lo cual se hace la consulta en las listas restrictivas y de control.

- Según la Circular Externa 100-000006 de 2016 [23], la única lista vinculante para Colombia es la lista ONU, pero, para tener un mayor control, es recomendable que la empresa consulte antecedentes en las listas disponibles (Policía Nacional, Procuraduría, OFAC, etc.) y elabore, 
alimente y consulte listas de control propias que permitan identificar si algún interesado en vínculos comerciales cuenta con antecedentes o reportes relacionados con el LA/FT.

- Principio "Conozco el mercado", permite conocer el entorno en que se desempeña la empresa.

- Instrumentos para detección de operaciones inusuales, lo cuales deben contener tipologías de operaciones de LA/FT, señales de alerta y procedimientos para el análisis y reporte de operaciones inusuales y sospechosas.

\subsection{ETAPA 5: DIVULGACIÓN Y DOCUMENTACIÓN}

Consiste en proporcionar un sistema de documentación que permita divulgar los procedimientos, instructivos, manuales y programas de capacitación establecidos por la empresa.

- PASO 1: proveer un sistema de documentos y registros de las etapas y elementos del sistema de gestión del riesgo de LA/FT. Es necesario definir los responsables de la documentación del sistema de gestión del riesgo de LA/FT, el cual debe comprender:

- Procedimientos e instructivos que soportan la implementación de las metodologías del SAGRLAFT.

- Registros documentales que soportan todo lo relacionado con las contrapartes, las operaciones inusuales y las operaciones sospechosas.

- Informes de los órganos de control.

- Los documentos que soportan los planes de acción implementados con el fin de mejorar los controles.

- Los documentos que soportan los requerimientos de información realizados por los órganos de control con sus respectivas respuestas.

- Soportes de los análisis de las operaciones inusuales y sospechosas.

- Procesos disciplinarios realizados por incumplimientos del SAGRLAFT.

- Planes y programas de capacitación del SAGRLAFT.

- Reportes internos y externos del SAGRLAFT.

- PASO 2: definir procedimientos para la realización de reportes internos y externos. Los reportes externos son los reportes que se hacen a la UIAF de operaciones sospechosas (ROS) e intentadas. Los reportes internos constan de:

- Transacciones inusuales: generación de señales de alerta.

- Reportes de operaciones sospechosas.

- Reportes en la etapa de seguimiento o monitoreo: producto del resultado del análisis de una operación inusual.

- PASO 3: diseño y ejecución del programa de capacitación y del plan de divulgación del sistema de gestión del riesgo de LA/FT. El programa de capacitación debe cumplir con las siguientes condiciones:

- Debe tener una frecuencia anual, cuyo contenido debe variar y estar actualizado.

- Se deben evaluar las capacitaciones dadas con el fin de comprobar el alcance de los objetivos propuestos.

- Definir la forma para ejecutar el programa de capacitación.

- PASO 4: divulgación de los controles para mitigar el riesgo del LA/FT. El plan de comunicación y divulgación con los interesados externos consiste en informar; a través de los formatos utilizados con las contrapartes, sobre la adopción del SAGRLAFT y los compromisos adquiridos por quienes tienen o pretenden tener relaciones contractuales con la empresa. En el proceso de inducción se deben divulgar las políticas y procedimientos establecidos por la empresa sobre el SAGRLAFT y a su vez dejar constancia de que los funcionarios fueron capacitados y se han comprometido con su cumplimiento.

- PASO 5: definir los procedimientos para la imposición de sanciones ante el incumplimiento de la aplicación de controles. Cada empleado de la entidad empresarial se debe comprometer con la observación de las funciones y procedimientos establecidos para impedir la utilización de la empresa en actividades de LA/FT, ya sea de manera directa o indirecta por medio de sus operaciones. Si se incumplen los códigos de comportamiento, normativas o estatutos procedimentales que indica el SAGRLAFT, la empresa se verá obligada a aplicar las sanciones o amonestaciones disciplinarias que correspondan. 
Operación del sistema de autocontrol y gestión del riesgo de lavado de activos y financiación del terrorismo en empresas del sector comercial Operation of the system for self-control and management of the risk of money laundering and financing of terrorism in companies of the commercial sector

\subsection{ETAPA 6: SEGUIMIENTO O MONITOREO}

Consiste en el monitoreo de los riesgos y en la medición de efectividad de los planes de gestión de ellos, del enfoque administrativo y el sistema de seguimiento a la aplicación de la normativa. Para desarrollar un proceso de seguimiento o de monitoreo efectivo se deben definir procedimientos que permitan verificar que los controles son óptimos y que son aplicados a todos los factores y eventos de riesgo.

Con un monitoreo y control eficiente es posible detectar operaciones que generen alarmas relacionadas con LA/FT, las cuales no son acordes al perfil del cliente, asociado, empleado, proveedor, PEP o negociación y requieren de un análisis para determinar el tipo de operación y el reporte de la operación sospechosa (ROS). El modelo de gestión resumido se describe en la figura 2; los pasos más importantes en este modelo corresponden al diagnóstico, identificación de riesgos y la adopción de controles. En estos pasos es donde se debe implementar la matriz de riesgo definida en la etapa 4 para efectuar la correcta identificación de los procesos en riesgo, los riesgos asociados y los controles que serán aplicados para mitigarlos.

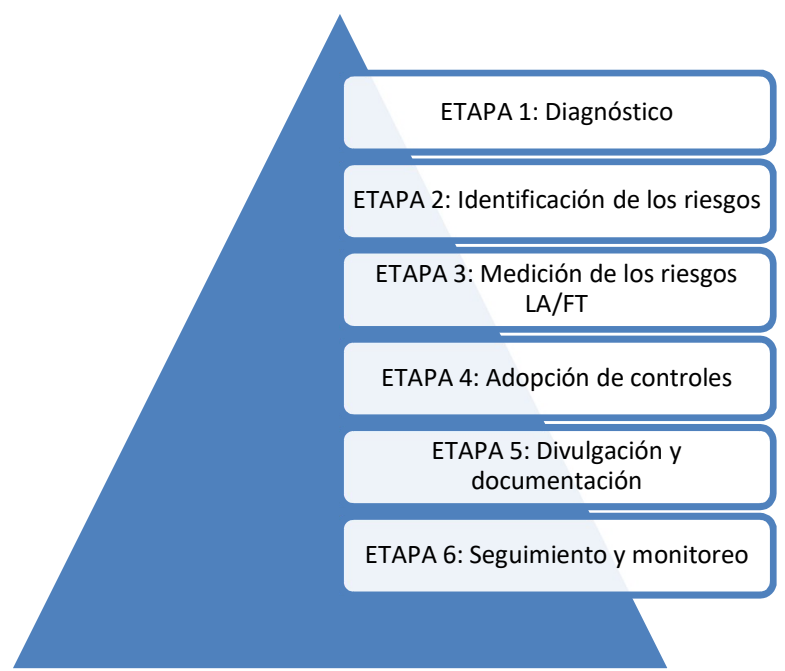

Figura 1. Estructura del Sistema de Gestión de Riesgo de LA/FT

\section{CONCLUSIONES}

El lavado de activos y la financiación del terrorismo afectan a todos los sectores económicos, por lo cual con la implementación del SAGRLAFT se beneficia la sociedad en general puesto que el Gobierno y las empresas actúan en conjunto en la prevención y el control del LA/FT y no se descarga la responsabilidad solo al sector financiero.

El Sistema de Autocontrol y Gestión de riesgo LA/FT no cuenta con un único modelo válido ante la Superintendencia de Sociedades ya que cada compañía debe diseñar su propio modelo de acuerdo con las características de la empresa y los factores de riesgo identificados. La adopción de mecanismos e instrumentos que permitan evitar la materialidad de eventos de riesgo de LA/FT en la empresa también evita que deba asumir los riesgos asociados al LA/FT; riesgo reputacional, legal, de contagio y operacional, de tal forma que pueda gozar de perdurabilidad en el tiempo y de buena imagen.

La lucha contra la financiación del terrorismo y el lavado de activos no depende únicamente de la expedición de la norma, ya que solo este hecho no es garantía de que se mitigue el riesgo de LA/FT, se requiere que cada empresa adopte estándares internacionales, acate las normas contra el LA/FT, se base en experiencias propias y ajenas para que evite ser utilizada para dar apariencia de legalidad a dineros provenientes de actividades ilícitas y/o de financiar al terrorismo directa e indirectamente.

Es importante aplicar metodologías, procesos y hacer uso de herramientas que permitan conocer a los clientes, proveedores, asociados, socios, empleados directos e indirectos, personas públicamente expuestas y los negocios virtuales o presenciales, para evitar el inicio de relaciones contractuales con personas naturales o jurídicas involucradas con actividades de LA/FT, como también es necesario que las empresas dispongan de herramientas tecnológicas que permitan detectar oportunamente operaciones poco usuales y bajo sospecha que ameriten el análisis y reporte, según la situación. 
En definitiva, en Colombia se ha respaldado lo concerniente a combatir el LA/FT y prevenir que se legalicen los recursos económicos provenientes del ejercicio de dichas actividades delictivas, pero se requiere el compromiso de todo el sector comercial para continuar con la gestión eficiente de dichos riesgos.

\section{REFERENCIAS}

[1] J. P. Rodríguez, I. E. Galvis, Aproximación a los instrumentos administrativos para la prevención y el control del lavado de activos en Colombia, Cuadernos de Contabilidad, 9 (24), 2008.

[2] A. Chatain, Pierre-Laurent Herndez-Coss, R. Borowik, K. Zerzan. Integrity in Mobile Phone Financial Services: Measures for Mitigating the Risks from Money Laundering and Terrorist Financing, The World Bank, https://doi.org/10.1596/978-0-8213-7556-3, 2008.

[3] N. Beekarry, International Anti-Money Laundering and Combating the Financing of Terrorism Regulatory Strategy: A Critical Analysis of Compliance Determinants in International Law. Nw. J. Int'I L. \& Bus., 2011, 31, 137.

[4] M. Levi, T. Halliday, P. Reuter, Global surveillance of dirty money: Assessing assessments of regimes to control money-laundering and combat the financing of terrorism, 2014.

[5] G. Merlonghi, "Fighting financial crime in the age of electronic money: opportunities and limitations", Journal of Money Laundering Control, Vol. 13 Issue: 3, 2010, pp. 202-214, https://doi.org/10.1108/13685201011057118

[6] S. Haigner, F. Schneider, F. Wakolbinger, Combating money laundering and the financing of terrorism: A survey. Economics of Security Working Paper, 2012.

[7] A. Y. Shehu. Promoting financial inclusion for effective anti-money laundering and counter financing of terrorism (AML/CFT). Crime, Law and Social Change, 57(3), 2012, 305-323. https://doi.org/10.1007/s10611-0119351-0

[8] D. Chaikin, A Critical Analysis of the Effectiveness of Anti-Money Laundering Measures with Reference to Australia. The Palgrave Handbook of Criminal and Terrorism Financing Law Springer, 2018, pp. 293-316.

[9] N. Omar, Z. A. Johari, R. Arshad, Money Laundering - FATF Special Recommendation VIII: A Review of Evaluation Reports. INTERNATIONAL CONFERENCE ON CORPORATE GOVERNANCE \& STRATEGIC MANAGEMENT (ICGSM) 2014, 145, 2014, pp. 211-225. https://doi.org/10.1016/j.sbspro.2014.06.029

[10] Superintendencia de Sociedades Prevención y gestión del riesgo de LA/FT, 2016. Recuperado de http://www.supersociedades.gov.co/asuntos-economicos-y-contables/estudios-y-supervision-por-

riesgos/prevencion-riesgo-lavado-de-activos/capacitacion/Documents/EE3-

\%20Presentaci\%C3\%B3n\%20LAFT\%20nueva\%20circular-\%202016\%20XI\%2017.pptx

[11] S. Rivero Mejía, J. Ramírez Canedo, La relación universidad - empresa - estado en el ámbito colombiano y el proceso de gestión tecnológica. Revista CINTEX, 16, 2011, pp. 96-102. Recuperado de http://www.pascualbravo.edu.co:5056/cintexpb/index.php/cintex/article/view/88

[12] J. Echavarría, Condiciones Laborales y Productivas en Microempresas de Confección de la ciudad de Medellín. Revista CINTEX, 20(2), 2015, pp. 79-95. Recuperado de http://www.pascualbravo.edu.co:5056/cintexpb/index.php/cintex/article/view/25

[13] R. Barry Johnston, O. M. Nedelescu, The impact of terrorism on financial markets. Journal of Financial Crime, 13(1), 2006, p. p7-25.

[14] G. Y. Mariño Becerra, F. D. J. Chaparro Gómez, I. Medina Sandoval, Determinants of Risk Prevention for Money Laundering and Terrorist Financing (ML/TF) in Real Sector. AD-minister, (25), 2014, pp. 7-35.

[15] V. Mitsilegas, B. Gilmore, The EU legislative framework against money laundering and terrorist finance: a critical analysis in the light of evolving global standards. International and comparative law quarterly, 56(01), 2007, pp. 119-140.

[16] Superintendencia Financiera de Colombia, Circular Externa No. 022 de 2007, 2007.Recuperado de http://www.sarlaft.com/html/Capitulo_11.pdf

[17] M Montoya. El lavado de activos obstáculo para el desarrollo, 2008. Recuperado de http://www.seminariopublica.info/maindata/seminario/200824-183411/imagesdirs/Mauoricio\%20Montoya.pdf.

[18] FATF, National Money Laundering and Terrorist Financing Risk Assessment. Paris: Financial Action Task Force, 2013.

[19] N. Gilmour, T. Hicks, S. Dilloway, Examining the practical viability of internationally recognised standards in preventing the movement of money for the purposes of terrorism: A crime script approach. Journal of Financial Crime, 24(2), 2017, pp. 260-276. https://doi.org/10.1108/JFC-04-2016-0027 
Operación del sistema de autocontrol y gestión del riesgo de lavado de activos y financiación del terrorismo en empresas del sector comercial Operation of the system for self-control and management of the risk of money laundering and financing of terrorism in companies of the commercial sector

[20] L. Koker, aligning anti-money laundering, combating of financing of terror and financial inclusion: Questions to consider when FATF standards are clarified. Journal of Financial Crime, 18(4), 2011, pp. 361-386. https://doi.org/10.1108/13590791111173704

[21] A. S. M. Irwin, K.-K. R. Choo, L. Liu. An analysis of money laundering and terrorism financing typologies. Journal of Money Laundering Control, 15(1), 2011, pp.85-111. https://doi.org/10.1108/13685201211194745

[22] UNODC y Cámara de Comercio de Bogotá, Modelo de Gestión del Riesgo de LA/FT para el sector real: guía para la adopción de un Sistema de Gestión del Riesgo de Lavado de Activos y de Financiación del Terrorismo en el sector real, 2013.

[23] SUPERINTENDENCIA DE SOCIEDADES, CIRCULAR EXTERNA 100-000006. Sistema de Autocontrol y Gestión del Riesgo de LA/FT. Reporte Obligatorio de información a la UIAF. 2016.

[24] J. J. Castro Maldonado, J. A. Patiño Murillo, and C. Gómez López, "Procesos de I+D+i en el Centro de Servicios y Gestión Empresarial del Servicio Nacional de Aprendizaje SENA 2015-2017," Revista ESPACIOS, vol. 39, no. 20, p. 21, 2018. 